\title{
Cooperative OFDM Channel Estimation with Frequency Offsets
}

\author{
Zhongshan Zhang, Wei Zhang and Chintha Tellambura \\ Department of Electrical and Computer Engineering \\ University of Alberta, Edmonton, Alberta, Canada T6G 2V4 \\ \{zszhang,wzhang, chintha\}@ece.ualberta.ca
}

\begin{abstract}
This paper discusses channel estimation in a cooperative orthogonal frequency-division multiplexing (OFDM) network in the presence of frequency offsets. Both the amplifyand-forward (AF) and decode-and-forward (DF) relaying modes are analyzed. In order to eliminate the multiple access interference (MAI), the maximum number of active $A F$ and DF relays are $\left\lfloor\frac{N}{2 L-1}\right\rfloor$ and $\left\lfloor\frac{N}{L}\right\rfloor$, respectively, where $N$ is the total number of subcarriers, $L$ is the channel order and $\lfloor a\rfloor$ is the maximum integer part of $a$. The pairwise error probability (PEP) of orthogonal space-time coding in cooperative OFDM due to both the frequency offset and channel estimation errors is also evaluated. The optimal power allocation ratio between the source and the set of the relays to minimize the PEP is derived for both the relay modes. When $L<16$, the DF mode always outperforms the AF mode in terms of PEP. Given 16 active relays and with $L=4$, the proposed DF mode outperforms the AF mode by about $9 \mathrm{~dB}$ for a frequency offset error variance of $10^{-3}$, and this gap increases to about $11.3 \mathrm{~dB}$ when the variance increases to $10^{-2}$.
\end{abstract}

\section{INTRODUCTION}

Cooperative diversity, in which multiple nodes cooperate to form a virtual multi-antenna array, has been intensively studied as a form of spatial diversity [1]. Although current research on cooperative networks mainly focuses on single-carrier (SC) systems [2], relay networks can also be implemented for orthogonal frequency-division multiplexing (OFDM) [3]. However, the effect of frequency offset, which degrades the Signalto-Interference-plus-Noise Ratio (SINR), is not considered in [3]. The effect of the frequency offset on cooperative OFDM or OFDM access (OFDMA) uplink transmission is studied in [4], [5]. The performance degradation of a cooperative OFDMA uplink due to the frequency offset is analyzed in [6].

Channel estimation errors can significantly impact the system performance. The amplify-and-forward (AF)-SC Channel estimation is discussed in [7]. By using Alamouti-coded pilot symbols, [8] provides synchronization and channel estimation for a cooperative OFDM system. However, the optimal channel estimation in cooperative OFDM in the presence of frequency offsets is still an open issue.

This paper investigates the optimal channel estimation for a cooperative AF or decode-and-forward (DF) OFDM system in the presence of frequency offsets. We show that the optimal channel estimation requires the multiple access interference (MAI) among the relays be eliminated and that the maximum number of active AF and DF relays be $\left\lfloor\frac{N}{2 L-1}\right\rfloor$ and $\left\lfloor\frac{N}{L}\right\rfloor$, respectively, where $N$ is the total number of subcarriers, $L$ is the maximum channel order, and $\lfloor a\rfloor$ represents the maximum integer part of $a$. Based on the SINR analysis, the pairwise error probability (PEP) of the orthogonal space-time coded system is evaluated.

This paper is organized as follows. Section II introduces the cooperative OFDM uplink system. Optimal channel estimation is analyzed in Section III. The PEP of the orthogonal space-time coded system is derived in Section IV. Section V discusses the numerical results. Finally, Section VI concludes the paper.

Notation: $(\cdot)^{T},(\cdot)^{H},(\cdot)^{-1}$ and $(\cdot)^{*}$ denote the transpose, conjugate transpose, inverse and complex conjugate. The imaginary unit is $\jmath=\sqrt{-1}$. $(\cdot)^{*}$ denotes the complex conjugate. A circularly symmetric complex Gaussian variable with mean $a$ and variance $\sigma^{2}$ is denoted by $z \sim \mathcal{C N}\left(a, \sigma^{2}\right) . \mathbf{x}[i]$ represents the $i$-th element of vector $\mathbf{x} .[\mathbf{A}]_{i j}$ represents the $i j$-th element of matrix $\mathbf{A}$. The $N \times N$ identity matrix is $\mathbf{I}_{N}$. The $M \times N$ all-zero matrix is $\mathbf{O}_{M \times N} \cdot \operatorname{diag}\{\mathbf{x}\}$ represents a diagonal matrix with its $n$-th diagonal element $\mathbf{x}[n]$.

\section{Cooperative OFDM Signal Model}

The cooperative OFDM system is shown in Fig. 1. In the AF mode, all the relays simply amplify and retransmit the received signal to the destination. In the DF mode, the relays first demodulate and decode the received symbol, and only the relays without decoding errors will re-encode and retransmit the received signal to $D$.

\section{A. Channel Model}

In this paper, $S$ represents the source node and $R_{k}, k \in$ $\{1, \cdots, M\}$, represents the $k$-th relay, where $M \geq 1$ is the total number of relays. The time-invariant composite channel impulse response between node $a$ and node $b$ is modelled as

$$
h_{a, b}(\tau)=\sum_{l=0}^{L-1} h_{a, b}[l] \delta\left(\tau-l T_{s}\right),
$$

where $h_{a, b}[l]$ is the channel gain between nodes $a$ and $b$ at the $l$-th tap, and $\delta(x)$ is the unit impulse function. $L$ is the maximum channel order for any pair of nodes, and $T_{s}=$ $1 / B$ with $B$ representing the total bandwidth. Accordingly, the discrete $T_{s}$-spaced channel impulse response between nodes $a$ and $b$ is $\tilde{\mathbf{h}}_{a, b}=\left[h_{a, b}(0), h_{a, b}(1), \cdots, h_{a, b}(L-\right.$ $1)]^{T}$. The frequency-domain channel coefficient matrix is 


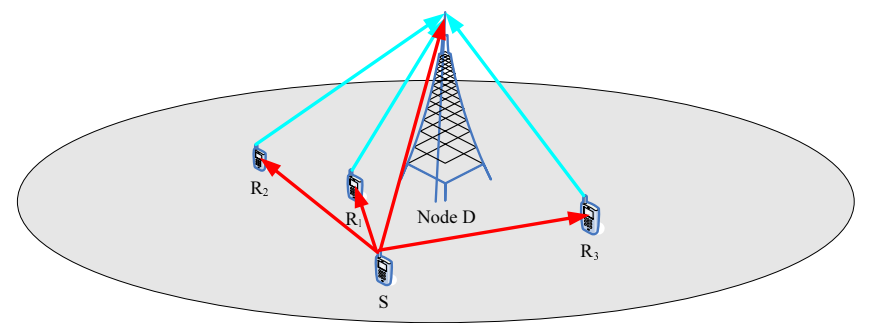

(a) Cooperative OFDM Transmission

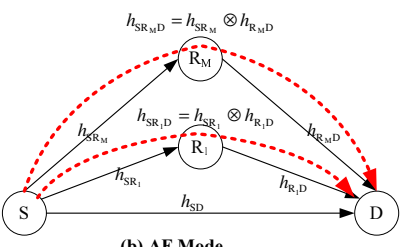

(b) AF Mode

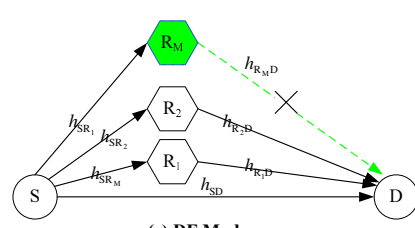

(c) DF Mode

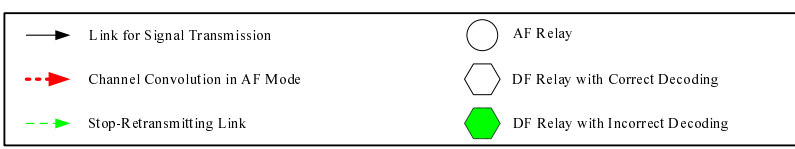

Fig. 1. Cooperative OFDM uplink transmission (a) Cooperative Group organization (b) Virtual tunnel created by cooperative relay (c) Relay selection in cooperative OFDM uplink transmission.

$\mathbf{H}_{a, b}=\operatorname{diag}\left\{H_{a, b}[0], \cdots, H_{a, b}[N-1]\right\}$, where $H_{a, b}[n]=$ $\sum_{d=0}^{L-1} h_{a, b}(d) e^{-\frac{\partial 2 \pi n d}{N}}$ is the channel frequency response on the $n$-th subcarrier.

The channel gains $h_{a, b}(l)$ are modelled as complex Gaussian zero-mean random variables (RVs). The $l$-th tap for the $S \rightarrow R_{k}$ channel has variance $\sigma_{R, S}^{2}(i)$, which is independent of $k$, and $\sum_{i=0}^{L-1} \sigma_{R, S}^{2}(i)=1$. The $S \rightarrow D$ and $R_{k} \rightarrow D$ channels are modelled similarly, with $h_{D, S}(i) \sim \mathcal{C N}\left(0, \sigma_{D, S}^{2}(i)\right)$ and $h_{D, R_{k}}(i) \sim \mathcal{C N}\left(0, \sigma_{D, R}^{2}(i)\right)$ for each $1 \leq k \leq M$. We assume that an independent and identically distributed (i.i.d.) statistics is assumed to each channel between the mobile node and the base station, and that $\sum_{i=0}^{L-1} \sigma_{D, S}^{2}(i)=\sum_{i=0}^{L-1} \sigma_{D, R}^{2}(i)=\mathcal{L}_{u}$, where $0<\mathcal{L}_{u}<1$ represents large-scale fading coefficient.

\section{B. OFDM Signal Model}

The input data bits are first mapped to complex symbols drawn from a typical signal constellation such as phase-shift keying (PSK) or quadrature amplitude modulation (QAM). An $N \times 1$ vector $\tilde{\mathbf{X}}_{S}=\left[X_{S}[0], X_{S}[1], \cdots, X_{S}[N-1]\right]=$ $\tilde{\mathbf{X}}_{S}^{d}+\tilde{\mathbf{X}}_{S}^{p}$ is used to represent these symbols sent by the node $S$, where $\tilde{\mathbf{X}}_{S}^{d}$ and $\tilde{\mathbf{X}}_{S}^{p}$ are the $N \times 1$ data and pilot vectors, respectively. Discrete signal samples are generated by taking the Inverse Discrete Fourier Transform (IDFT) of $\tilde{\mathbf{X}}_{S}$. A length$L_{C P}$ cyclic prefix (CP) is appended before transmission, and inter-symbol interference (ISI) can be avoided if $L_{C P}>L$.

The proposed cooperative transmission can be performed within two time slots. The time slot is conceptually inter- changeable with the OFDM symbol. In the first time slot, the $S \rightarrow D$ and $S \rightarrow R_{k}$ transmissions take place, and $R_{k} \rightarrow D$ transmissions will happen in the second time slot.

\section{First Time Slot}

The received signal at node $D$ or relay $R_{k}$ is

$$
\mathbf{y}_{z, 1}=\sqrt{\alpha \bar{P}} \mathbf{E}_{z, S} \mathbf{F} \mathbf{H}_{z, S} \tilde{\mathbf{X}}_{S}+\mathbf{w}_{z, 1},
$$

where $z \in\left\{D, R_{k}\right\}, 0<\alpha<1$ represents the power allocation ratio between the source node and the set of relays, $\bar{P}$ stands for the average power of each subcarrier, $\varepsilon_{z, S}$ is the normalized frequency offset between nodes $S$ and $z$, $\mathbf{w}_{z, 1}$ is an additive white Gaussian noise (AWGN) vector with $w_{z, 1}(n) \sim \mathcal{C N}\left(0, \sigma_{w}^{2}\right)$, and the IDFT matrix $\mathbf{F}$ is defined as $[\mathbf{F}]_{n k}=\frac{1}{\sqrt{N}} e^{\frac{22 \pi n k}{N}}$ for $0 \leq n, k \leq N-1$. The frequency-offset dependent matrix $\mathbf{E}_{a, b}$ is defined by $\mathbf{E}_{a, b}=\operatorname{diag}\left\{1, e^{\frac{j 2 \pi \varepsilon_{a, b}}{N}}, \cdots, e^{\frac{j 2 \pi \varepsilon_{a, b}(N-1)}{N}}\right\}$. Note that (2) is valid for both the $\mathrm{AF}$ and $\mathrm{DF}$ modes.

The post-DFT demodulator outputs can be represented as

$$
\begin{aligned}
\mathbf{r}_{z, 1}= & \mathbf{F}^{H} \mathbf{y}_{z, 1}=\underbrace{\sqrt{\alpha N \bar{P}} \mathbf{E}_{z, S}^{\mathrm{cir}} \mathbf{X}_{S}^{p} \mathbf{F}_{(L)}^{H}}_{\mathbf{P}_{z, S}(N \times L)} \tilde{\mathbf{h}}_{z, S} \\
& +\sqrt{\alpha N \bar{P}} \mathbf{E}_{z, S}^{\mathrm{cir}} \mathbf{X}_{S}^{d} \mathbf{F}_{(L)}^{H} \tilde{\mathbf{h}}_{z, S}+\mathbf{F}^{H} \mathbf{w}_{z, 1},
\end{aligned}
$$

where $z=\left\{D, R_{k}\right\}, \mathbf{E}_{a, b}^{\mathrm{cir}}=\mathbf{F}^{H} \mathbf{E}_{a, b} \mathbf{F}, \mathbf{F}_{(L)}$ is the first $L$ rows of $\mathbf{F}$, and $\mathbf{X}_{S}=\operatorname{diag}\left\{\tilde{\mathbf{X}}_{S}^{d}\right\}+\operatorname{diag}\left\{\tilde{\mathbf{X}}_{S}^{p}\right\}$.

\section{Second Time Slot}

In the second time slot, the relays will forward the received signal from $S$ by using the total power $(1-\alpha) N \bar{P}$, and an identical power is allocated to each relay.

1) AF Mode: The received symbol at $D$ is

$$
\mathbf{y}_{D, 2}^{\mathrm{AF}}=\rho_{R} \sqrt{\frac{(1-\alpha) \bar{P}}{M}} \sum_{k=1}^{M} \mathbf{E}_{D, R_{k}} \mathbf{F H}_{D, R_{k}} \mathbf{r}_{R_{k}, 1}+\mathbf{w}_{D, 2} \text {, }
$$

where $\rho_{R}=\left(\alpha \bar{P}+\sigma_{w}^{2}\right)^{-\frac{1}{2}}$ is the amplifying coefficient.

2) DF Mode: In the DF mode, each relay first demodulates and decodes the received symbol, but only the relays without a decoding error will re-encode this symbol and retransmit it. We assume that $m$ out of $M$ relays can decode correctly, where $0 \leq m \leq M$, and the received symbol at node $D$ is

$$
\mathbf{y}_{D, 2}^{\mathrm{DF}}=\sqrt{\frac{(1-\alpha) \bar{P}}{m}} \sum_{k=1}^{m} \mathbf{E}_{D, R_{k}} \mathbf{F H}_{D, R_{k}} \tilde{\mathbf{X}}_{R_{k}}+\mathbf{w}_{D, 2} .
$$

After performing the DFT on $\mathbf{y}_{D, 2}^{\mathrm{DF}}$, the demodulated signal is

$$
\begin{aligned}
\mathbf{r}_{D, 2}^{\mathrm{DF}} & =\mathbf{F}^{H} \mathbf{y}_{D, 2}^{\mathrm{DF}}=\sum_{k=1}^{m} \underbrace{\sqrt{\frac{(1-\alpha) N \bar{P}}{m}} \mathbf{E}_{D, R_{k}}^{\mathrm{cir}} \mathbf{X}_{R_{k}}^{p} \mathbf{F}_{(L)}^{H}}_{\mathbf{P}_{D, R_{k}}^{\mathrm{DF}}} \tilde{\mathbf{h}}_{D, R_{k}} \\
& +\sum_{k=1}^{m} \sqrt{\frac{(1-\alpha) N \bar{P}}{m}} \mathbf{E}_{D, R_{k}}^{\mathrm{cir}} \mathbf{X}_{R_{k}}^{d} \mathbf{F}_{(L)}^{H} \tilde{\mathbf{h}}_{D, R_{k}}+\mathbf{F}^{H} \mathbf{w}_{D, 2} .
\end{aligned}
$$




\section{Channel Estimation for Cooperative OFDM}

In the first time slot, the destination receives from the source only, and hence there is no MAI. However, in the second time slot, the destination receives the signal from multiple relays and MAI occurs. Optimal channel estimation requires that the MAI be totally eliminated at the destination.

\section{A. Optimal Pilot to Eliminate MAI in The Second Time Slot}

Since an identical pilot, i.e., $\mathbf{X}_{S}^{p}$, is received at each relay in the first time slot, the received pilot at the destination $D$ in the second time slot is also $\mathbf{X}_{S}^{p}$ if the relays simply retransmit the received signal without modifying it. In this case, node $D$ will not be able to identify $\tilde{\mathbf{h}}_{D, R_{k}, S}$ in the AF mode (or $\tilde{\mathbf{h}}_{D, R_{k}}$ in the DF mode) for each $k$.

Optimal pilot proposed in [9] can be readily adapted here to mitigate the MAI in the second time slot. Without loss of generality, we assume that the retransmitted pilot for the relay $R_{k}$ is $\mathbf{X}_{R_{k}}^{p}$. In the DF mode, each relay can optimize its own pilot before its retransmission. However, in the AF mode, only an identical pilot can be retransmitted unless each relay does some modification to its received pilot.

1) AF Mode: A modified AF relaying mode is applied in each relay to mitigate the MAI. With this modified AF mode, the received signal $\mathbf{y}_{R_{k}, 1}$ in the $k$-th relay $R_{k}$ is multiplied by a pre-modulation matrix $\Pi_{k}$. The received signal at $D$ is demodulated as in (7), where $\mathbf{X}_{R_{k}}^{p}$ is a unique pilot of node $k,{ }^{1} Q_{1}(\alpha)=\sqrt{\frac{\alpha(1-\alpha) N \bar{P}^{2}}{M\left(\alpha \bar{P}+\sigma_{w}^{2}\right)}}, Q_{2}(\alpha)=\sqrt{\frac{(1-\alpha) N \bar{P}}{M\left(\alpha \bar{P}+\sigma_{w}^{2}\right)}}$, $\mathbf{E}_{D, R_{k}, S}^{\mathrm{cir}}=\mathbf{E}_{D, R_{k}}^{\mathrm{cir}} \mathbf{E}_{R_{k}, S}^{\mathrm{cir}}=\mathbf{E}_{D, S}^{\mathrm{cir}}, \mathbf{W}_{\eta}=\operatorname{diag}\left\{\mathbf{F}^{H} \mathbf{w}_{R_{k}, 1}\right\}$, $\tilde{\mathbf{h}}_{D, R_{k}, S}=\left(\tilde{\mathbf{h}}_{R_{k}, S}^{T} \otimes \tilde{\mathbf{h}}_{D, R_{k}}^{T}\right)^{T}$, and $\otimes$ represents a convolutionary product operation.

Assuming that a total of $\mathcal{N}_{p}$ pilots are allocated per node (including the source and the relays) and that the pilot subcarriers, i.e., $\theta_{1}, \cdots, \theta_{\mathcal{N}_{p}}$, are identical for all the nodes. From [9], the optimal pilots to mitigate MAI is

$$
\begin{gathered}
{\left[\mathbf{X}_{R_{k}}^{p}\right]_{\theta_{i} \theta_{i}}=e^{\frac{j 2 \pi \theta_{i}(k-1)(2 L-1)}{N}}, \quad i=1, \cdots, \mathcal{N}_{p}} \\
\text { s.t. } \quad(2 L-1) M \leq \mathcal{N}_{p} \leq N, \quad N / \mathcal{N}_{p}=\text { integer; } \\
\theta_{i}(k-l)(2 L-1) / N \neq \text { integer, } \quad k \neq l ; \\
\theta_{2}-\theta_{1}=\cdots=\theta_{\mathcal{N}_{p}}-\theta_{\mathcal{N}_{p}-1}=N / \mathcal{N}_{p} .
\end{gathered}
$$

The matrix $\boldsymbol{\Pi}_{k}$ that satisfies $\mathbf{E}_{D, R_{k}}^{\mathrm{cir}} \boldsymbol{\Pi}_{k} \mathbf{E}_{R_{k}, S}^{\mathrm{cir}} \mathbf{X}_{S}^{p}=$ $\mathbf{E}_{D, S}^{\text {cir }} \mathbf{X}_{R_{k}}^{p}$ is resolved as

$$
\boldsymbol{\Pi}_{k}=\mathbf{E}_{R_{k}, S}^{\mathrm{cir}} \boldsymbol{\Lambda}_{k}\left(\mathbf{E}_{R_{k}, S}^{\mathrm{cir}}\right)^{-1}=\mathbf{F}^{H} \mathbf{E}_{R_{k}, S} \mathbf{F} \boldsymbol{\Lambda}_{k} \mathbf{F}^{H} \mathbf{E}_{R_{k}, S}^{-1} \mathbf{F},
$$

where $\boldsymbol{\Lambda}_{k}$ is a diagonal matrix with $\left[\boldsymbol{\Lambda}_{k}\right]_{\theta_{i} \theta_{i}}=$ $e^{\frac{j 2 \pi \theta_{i}(k-1)(2 L-1)}{N}}$, and $\left[\boldsymbol{\Lambda}_{k}\right]_{l l}=0$ for each $l \neq \theta_{i}$. Note that $\Pi_{k}$ does nothing to the data subcarriers.

Since in the AF mode, the channel order for all the $S \rightarrow$ $R \rightarrow D$ channels is $2 L-1$. From (8), the condition of $(2 L-$

\footnotetext{
${ }^{1}$ In the following analysis, we assume that a unique pilot is allocated to each relay, and each relay knows its own pilot, but how to perform this pilotallocation scheme is beyond the scope of this paper.
}

1) $M \leq \mathcal{N}_{p} \leq N$ must be satisfied for the optimal pilot design, and consequently $M \leq\lfloor N /(2 L-1)\rfloor$ should be satisfied.

2) DF Mode: In the DF mode, each correct-decoding relay will retransmit in the second time slot by modulating the pilot subcarriers with its own pilot but without changing the data subcarriers. The optimal pilot for $R_{k}$ in the DF mode is given by

$$
\begin{gathered}
{\left[\mathbf{X}_{R_{k}}^{p}\right]_{\theta_{i} \theta_{i}}=e^{\frac{j 2 \pi \theta_{i}(k-1) L}{N}}, \quad i=1, \cdots, \mathcal{N}_{p}} \\
\text { s.t. } L M \leq \mathcal{N}_{p} \leq N, \quad N / \mathcal{N}_{p}=\text { integer; } \\
\theta_{i}(k-l) L / N \neq \text { integer, } \quad k \neq l \\
\theta_{2}-\theta_{1}=\cdots=\theta_{\mathcal{N}_{p}}-\theta_{\mathcal{N}_{p}-1}=N / \mathcal{N}_{p} .
\end{gathered}
$$

Since the channel order for each $R \rightarrow D$ channel is $L$ in the DF mode, from (10), the condition of $L M \leq \mathcal{N}_{p} \leq N$ must be satisfied for the optimal pilot design, and we can easily conclude that $M \leq\lfloor N / L\rfloor$.

\section{Pairwise Error Probability (PEP) Analysis}

In this section, the pairwise error probability (PEP) of cooperative OFDM by considering both the frequency offset and channel estimation errors is derived. An orthogonal spacetime signal matrix $\overline{\mathbf{X}}_{S}=\left[\tilde{\mathbf{X}}_{S}(1), \tilde{\mathbf{X}}_{S}(2), \cdots, \tilde{\mathbf{X}}_{S}(T)\right]$, which is the $N \times T$ matrix, is assumed. The channel remains static over the code period.

\section{A. PEP for the AF Mode}

The probability that $\overline{\mathbf{X}}_{S}$ will be mistaken for another code $\overline{\mathbf{L}}_{S}$ is upper bounded by $\mathrm{P}_{\mathrm{r}}^{\mathrm{AF}}\left\{\overline{\mathbf{X}}_{S} \rightarrow \overline{\mathbf{L}}_{S} \mid 0<\alpha<1\right\}[10]$. In a high SINR regime with $\sigma_{e}^{2} \rightarrow 0$, the PEP can be approximated as in (11), where $\ell_{n}$ is the $n$-th eigenvalue of $\left(\overline{\mathbf{X}}_{S}-\overline{\mathbf{L}}_{S}\right)\left(\overline{\mathbf{X}}_{S}-\overline{\mathbf{L}}_{S}\right)^{H}$.

\section{B. PEP for the DF Mode}

In the DF mode, by using $P_{\text {relay }}$ to represent the average probability of decoding error at each relay, the probability that $m$ out of $M$ relays successfully decode the received signal is a Binomial distribution, i.e., $P_{\text {relay }, m}=\left(\begin{array}{c}M \\ m\end{array}\right)(1-$ $\left.P_{\text {relay }}\right)^{m} P_{\text {relay }}^{M-m}$. We also use $P_{S \rightarrow D}$ to represent the probability of the decoding error at $D$ in the first time slot. $P_{\text {relay }}$ and $P_{S \rightarrow D}$ are given by $P_{\text {relay }} \leq \prod_{n=0}^{L-1} \frac{1}{1+\frac{\bar{\gamma}_{R_{k} S, n} \ell_{n}}{4}}$ and $P_{S \rightarrow D} \leq \prod_{n=0}^{L-1} \frac{1}{1+\frac{\bar{\gamma}_{D S, n} \ell_{n}}{4}}$, respectively, where $\bar{\gamma}_{R_{k} S, n}$ represent the SINR of the $\stackrel{n}{S} \rightarrow R_{k}$ channel in the $n$-th tap.

In the second time slot, the $m$ relays with correct decoding will retransmit. The PEP that $\overline{\mathbf{X}}_{S}$ will be mistaken for another codeword $\overline{\mathbf{L}}_{S}$ is upper bounded by

$$
\mathrm{P}_{\mathrm{r}, m}^{\mathrm{DF}}\left\{\overline{\mathbf{X}}_{S} \rightarrow \overline{\mathbf{L}}_{S} \mid 0<\alpha<1\right\} \leq \prod_{n=0}^{L-1} \frac{1}{1+\frac{\bar{\gamma}_{D R, m, n} \ell_{n}}{4}},
$$




$$
\begin{aligned}
\tilde{\mathbf{r}}_{D, 2}^{\mathrm{AF}}= & \sum_{k=1}^{M} \underbrace{Q_{1}(\alpha) \overbrace{\mathbf{E}_{D, R_{k}}^{\mathrm{cir}} \mathbf{\Pi}_{k} \mathbf{E}_{R_{k}, S}^{\mathrm{cir}} \mathbf{X}_{S}^{p}}^{=\mathbf{E}_{(2 L-1)}^{\mathrm{cir}}, \mathbf{X}_{R_{k}}^{p}}}_{\tilde{\mathbf{P}}_{D, R_{k}, S}^{\mathrm{AF}}} \tilde{\mathbf{h}}_{D, R_{k}, S}^{H}+\sum_{k=1}^{M} Q_{2}(\alpha) \mathbf{E}_{D, R_{k}}^{\mathrm{cir}} \boldsymbol{\Pi}_{k} \mathbf{W}_{\eta} \mathbf{F}_{(L)}^{H} \tilde{\mathbf{h}}_{D, R_{k}}+\mathbf{F}^{H} \mathbf{w}_{D, 2} \\
& +\sum_{k=1}^{M} Q_{1}(\alpha) \mathbf{E}_{D, R_{k}}^{\mathrm{cir}} \mathbf{\Pi}_{k} \mathbf{E}_{R_{k}, S}^{\mathrm{cir}} \mathbf{X}_{S}^{d} \mathbf{F}_{(2 L-1)}^{H} \tilde{\mathbf{h}}_{D, R_{k}, S}
\end{aligned}
$$

$$
\lim _{\substack{\sigma_{2}^{2} \rightarrow 0 \\ \text { SNR } \rightarrow \infty}} \mathrm{P}_{\mathrm{r}}^{\mathrm{AF}}\left\{\overline{\mathbf{X}}_{S} \rightarrow \overline{\mathbf{L}}_{S} \mid 0<\alpha<1\right\} \leq\left(\frac{4\left[\frac{\mathcal{L}_{u}(M+1)(1-\alpha)}{\alpha}+1\right]}{\alpha(1-\alpha) \mathrm{SNR}}\right)^{2 L-1}\left(\frac{4}{\alpha \mathrm{SNR}}\right)^{L} \times\left(\prod_{n=0}^{2 L-2} \frac{M}{\left|\sum_{k=1}^{M} \tilde{\mathbf{h}}_{D, R_{k}, S}[n]\right|^{2} \ell_{n}}\right)\left(\prod_{n=0}^{L-1} \frac{1}{\left|\tilde{\mathbf{h}}_{D, S}[n]\right|^{2} \ell_{n}}\right)^{2}
$$

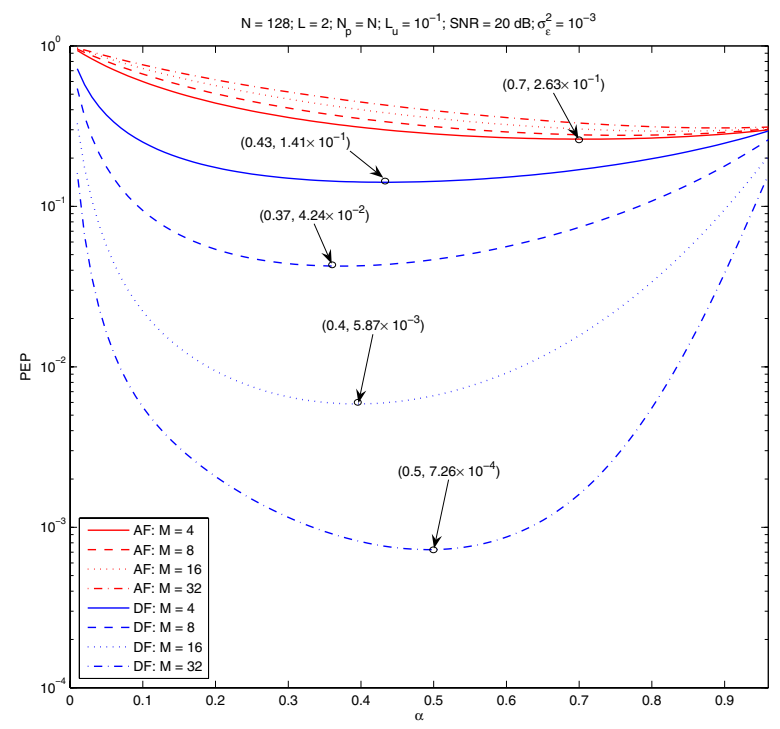

Fig. 2. PEP of the proposed cooperative transmission with $L=2$ and $\sigma_{e}^{2}=10^{-3}$.

where $\bar{\gamma}_{D R, m, n}$ represents the SINR of the $R \rightarrow D$ channel in the $n$-th multipath tap. Therefore, the averaged PEP of the DF mode is upper bounded by

$$
\overline{\mathrm{PEP}^{\mathrm{DF}}} \leq P_{S \rightarrow D} \sum_{m=0}^{M} P_{\text {relay }, m} \mathrm{P}_{\mathrm{r}, m}^{\mathrm{DF}}\left\{\overline{\mathbf{X}}_{S} \rightarrow \overline{\mathbf{L}}_{S} \mid 0<\alpha<1\right\} \text {. }
$$

\section{Numerical Results}

Uniform power-delay profiles are used between any pair of nodes; i.e., $\mathbb{E}\left\{\left|h_{R_{k}, S}(l)\right|^{2}\right\}=1 / L$ and $\mathbb{E}\left\{\left|h_{D, R_{k}}(l)\right|^{2}\right\}=$ $\mathbb{E}\left\{\left|h_{D, S}(l)\right|^{2}\right\}=\mathcal{L}_{u} / L$, where $l=0,1,2, \cdots, L-1$. We also assume that $N=128$.

The PEP performance of the proposed cooperative transmission in the presence of both the frequency offset and channel

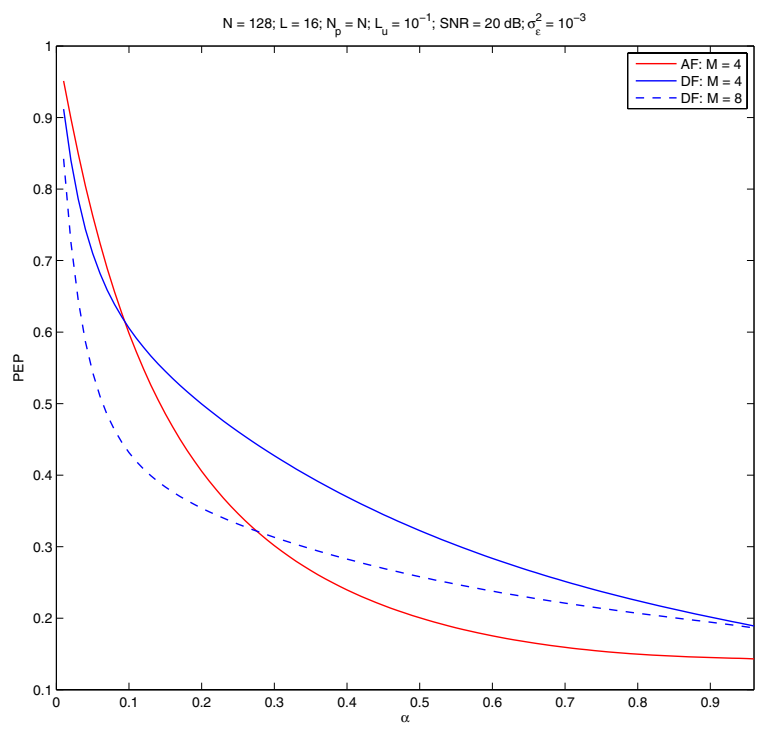

Fig. 3. PEP of the proposed cooperative transmission with $L=16$ and $\sigma_{e}^{2}=10^{-3}$.

estimation errors is illustrated in Figs.2-5. All the eigenvalues of $\left(\overline{\mathbf{X}}_{S}-\overline{\mathbf{L}}_{S}\right)\left(\overline{\mathbf{X}}_{S}-\overline{\mathbf{L}}_{S}\right)^{H}$ are set to 1 .

The PEP performance is compared between the AF and the DF modes for $L=2$ and $\sigma_{e}^{2}=10^{-3}$ in Fig. 2. Since the DF spatial diversity gain is proportional to the number of relays $M$, a larger number of active relays results in a lower PEP. In contrast, a larger number of AF relays results in a worse PEP performance. This difference can be explained as follows: In the AF mode, interference and noise accumulate in each relay, and this interference cannot be averaged out by using more relays. However, the DF mode eliminates the accumulation of interference and noise in the relays. For fixed $\sigma_{e}^{2}=10^{-3}$ but the channel order increasing to 16, the PEP performance is shown in Fig. 3. In this case, increasing the channel order always degrades the PEP performance in the DF mode because this mode can obtain only a $L$-order multipath diversity gain. 


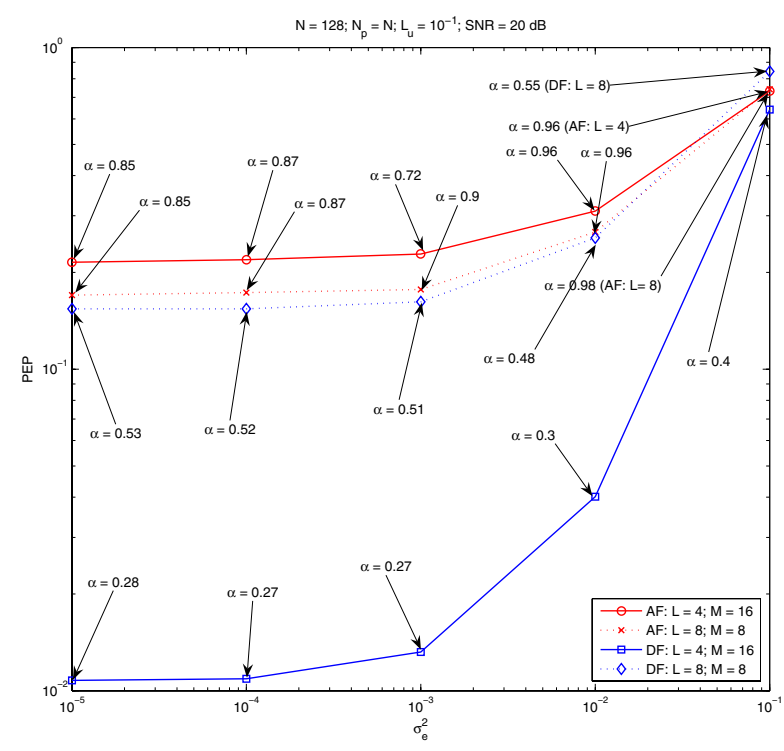

Fig. 4. PEP of the proposed cooperative transmission as a function of $\sigma_{e}^{2}$ with $L=4,8$.

The PEP performance as a function of $\sigma_{e}^{2}$ is shown in Fig. 4, which considers $(L=4, M=16)$ and $(L=8, M=8)$. For given relays, the DF mode always outperforms the AF mode for a small $\sigma_{e}^{2}$, although both modes approach the same PEP performance for large $\sigma_{e}^{2}$.

The PEP performances as functions of SNR are shown in Fig. 5 for $L=4, M=16, \sigma_{e}^{2}=10^{-2}$ and $10^{-3}$. The PEP performance of the DF mode is about $9 \mathrm{~dB}$ better than that of the AF mode at an error rate of $5 \times 10^{-3}$. As $\sigma_{e}^{2}$ increases to $10^{-2}$, the performance increases to about $11.3 \mathrm{~dB}$; i.e., the DF mode has a higher interference-mitigation capability than the AF mode.

\section{CONCLUSIONS}

Optimal channel estimation in a cooperative AF or DF OFDM in the presence of frequency offsets has been considered. For a given channel order $L$, the spatial diversity gain was much easier to obtain in the DF mode than in the AF mode. The PEP performance of an orthogonal spacetime code in the proposed cooperative transmission was also evaluated by taking into account both the frequency offset and channel estimation errors. For both relaying modes, a larger channel order always implies a higher interference and noise in relays, and this higher interference and noise degrades the SINR. Since a $(2 L-1)$-order multipath diversity gain can be obtained in the AF mode, a better PEP performance may be obtained as the channel order increases, provided that the channel order is not too large. Unlike the effects of increasing the channel order in the AF mode, increasing the channel order in the DF mode will always degrade the PEP performance. Interference will increase if more relays are used in the AF mode, resulting in degradation of performance. The

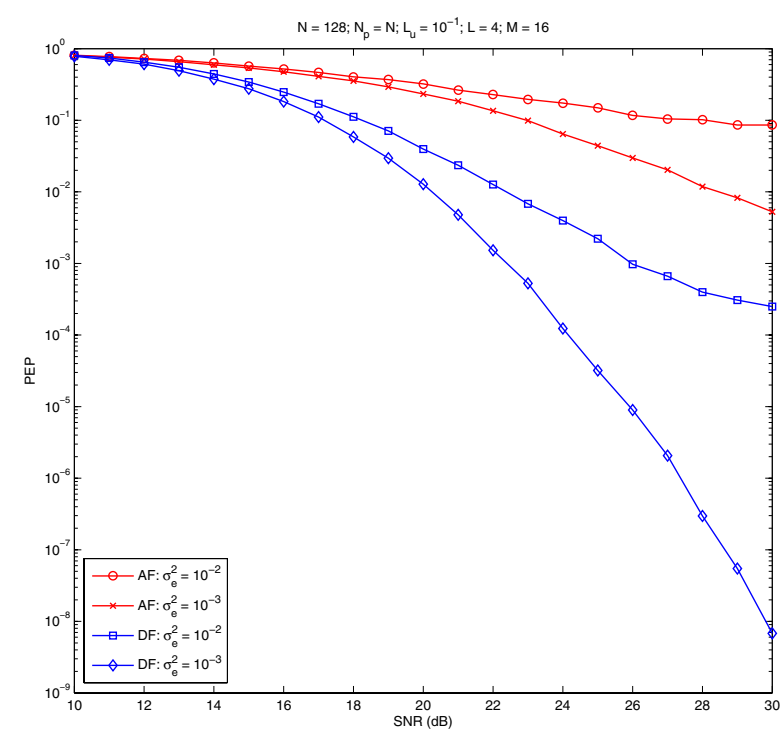

Fig. 5. PEP of the proposed cooperative transmission as a function of SNR with $L=4, M=16$ and $\sigma_{e}^{2}=10^{-2}, 10^{-3}$.

interference-mitigation capability of the DF mode brings a higher performance improvement to the DF mode by using more relays, and when the channel order is not too large, the DF mode always outperforms the AF mode in terms of PEP.

\section{REFERENCES}

[1] J. N. Laneman and G. W. Wornell, "Distributed space-time-coded protocols for exploiting cooperative diversity in wireless networks," IEEE Trans. Inform. Theory, vol. 49, no. 10, pp. 2415-2425, Oct. 2003.

[2] R. U. Nabar, H. Bolcskei, and F. W. Kneubuhler, "Fading relay channels: performance limits and space-time signal design," IEEE J. Select. Areas Commun., vol. 22, no. 6, pp. 1099-1109, Aug. 2004.

[3] B. Can, H. Yomo, and E. De Carvalho, "Hybrid forwarding scheme for cooperative relaying in OFDM based networks," in Proc. IEEE Int. Conf. Communications (ICC), vol. 10, Istanbul, June 2006, pp. 4520-4525.

[4] D. Sreedhar and A. Chockalingam, "ICI-ISI mitigation in cooperative SFBC-OFDM with carrier frequency offset," in IEEE Int. Symposium on Personal, Indoor and Mobile Radio Commun. (PIMRC), Athens, Sept. 2007, pp. 1-5.

[5] R. Raghunath and A. Chockalingam, "SIR analysis and interference cancellation in uplink OFDMA with large carrier frequency and timing offsets," in IEEE Wireless Commun. and Networking Conf., Kowloon, Mar. 2007, pp. 996-1001.

[6] Z. Zhang, C. Tellambura, and R. Schober, "Improved OFDMA uplink transmission via cooperative relaying in the presence of frequency offsets," IEEE Trans. Veh. Technol., To submit.

[7] C. Patel and G. Stuber, "Channel estimation for amplify and forward relay based cooperation diversity systems," IEEE Trans. Wireless Commun., vol. 6, no. 6, pp. 2348-2356, June 2007.

[8] O. S. Shin, A. M. Chan, H. T. Kung, and V. Tarokh, "Design of an OFDM cooperative space-time diversity system," IEEE Trans. Veh. Technol., vol. 56, pp. 2203-2215, July 2007.

[9] Z. Zhang, W. Zhang, and C. Tellambura, "MIMO-OFDM channel estimation in the presence of frequency offsets," IEEE Trans. Wireless Commun., vol. 7, no. 6, pp. 2329-2339, June 2008.

[10] H. Mheidat, M. Uysal, and N. Al-Dhahir, "Equalization techniques for distributed space-time block codes with amplify-and-forward relaying," IEEE Trans. Signal Processing, vol. 55, pp. 1839-1852, May 2007. 